\title{
Internet-Based Sex-Seeking Behavior Promotes HIV Infection Risk: A 6-Year Serial Cross-Sectional Survey to MSM in Shenyang, China
}

\author{
Shi Pan, ${ }^{1,2}$ Jun-Jie Xu, ${ }^{1,2}$ Xiao-Xu Han, ${ }^{1,2}$ Jing Zhang, ${ }^{1,2}$ Qing-Hai Hu, ${ }^{1,2}$ \\ Zhen-Xing Chu, ${ }^{1,2}$ Yan-Qiu Hai, ${ }^{1,2}$ Xiang Mao, ${ }^{1,2}$ Yan-Qiu Yu, ${ }^{1,2}$ Wen-Qing Geng, \\ Yong-Jun Jiang, ${ }^{1,2}$ and Hong Shang ${ }^{1,2}$ \\ ${ }^{1}$ Key Laboratory of AIDS Immunology of National Health and Family Planning Commission, Department of Laboratory Medicine, \\ The First Affiliated Hospital, China Medical University, Shenyang 110001, China \\ ${ }^{2}$ Collaborative Innovation Center for Diagnosis and Treatment of Infectious Diseases, Hangzhou, China
}

Correspondence should be addressed to Hong Shang; hongshang100@hotmail.com

Received 7 September 2016; Revised 16 November 2016; Accepted 1 December 2016

Academic Editor: Lei Zhang

Copyright (C) 2016 Shi Pan et al. This is an open access article distributed under the Creative Commons Attribution License, which permits unrestricted use, distribution, and reproduction in any medium, provided the original work is properly cited.

HIV prevalence is still rapidly increasing among Chinese men who have sex with men (MSM). The Internet also makes it easier for MSM to have casual partners. This study aims to evaluate the trend of Internet-based sex-seeking behavior of MSM and its impact on HIV prevalence, the distribution of HIV subtype strains, and transmitted drug resistance rates. A serial cross-sectional study was conducted from 2009 to 2014 . Of the 1,981 MSM, 50.5\% $(1,000 / 1,981)$ mainly sought homosexual partners through the Internet (Internet-based MSM, IBM). The proportion of IBM among total MSM subjects increased from $43.3 \%$ to $61.5 \%(p<0.001)$. HIV prevalence of IBM increased from $5.7 \%$ to $20.7 \%$, while that of non-Internet-based MSM (NIBM) increased from $7.0 \%$ to $14.7 \%$. A relative higher proportion of NIBM were infected with HIV CRF01_AE subtype than IBM (79.5\% versus 72.2\%, $p=0.52)$. Multivariable analysis found IBM had a significantly higher HIV prevalence than NIBM $(13.2 \%$ versus $10.5 \%$, aOR $=1.4,95 \%$ CI [1.0-1.9]). Being a migrant non-Shenyang resident MSM ( $\mathrm{aOR}=1.9,95 \% \mathrm{CI}[1.3-2.9])$ and occasionally/never using condoms with casual homosexual partners ( $\mathrm{aOR}=1.7,95 \%$ CI [1.1-2.6]) were two distinct risk factors for HIV infection in IBM. More efforts should be targeted towards developing interventions aimed at IBM, particularly migrant MSM and who engage in UAI with casual homosexual partners.

\section{Introduction}

Globally, men who have sex with men (MSM) suffer disproportionately with HIV infection. The prevalence of HIV in China continues to increase, with rates in Chinese MSM increasing from $0.9 \%$ to $7.3 \%$ from 2003 to 2013 [1]. Out of the newly reported HIV/AIDS cases in China in 2015, 28.3\% were from the MSM population [2], while in 2011, MSM only accounted for $13.7 \%$ of newly reported cases [3].

With the development of Internet and mobile technology, the ways of socializing and seeking homosexual partners have changed dramatically with MSM. More and more MSM use Internet- or mobile-based geosocial networking (GSN) applications to socialize and seek partners. The Internet makes it easier for MSM to seek casual homosexual partners. But Internet-based MSM [IBM] also have distinct characteristics which promotes their high-risk sexual behaviors [47]. As a result, there are great concerns for the potential effect of Internet-based socializing on HIV infection and transmission $[4,6]$.

The majority of published studies on IBM were completed via questionnaires done online or over the smart phone application [4-7]. These studies examined HIV testing and sexual behaviors and mainly found that IBM are usually younger, have higher educational level, and are more likely to test for HIV compared with non-Internet-based MSM [NIBM] [6-11]. A few studies compared the prevalence of HIV and sexually transmitted infections (STIs) between IBM 
and NIBM, but with inconclusive evidence on whether online partner-seeking increased the prevalence of HIV and or STIs $[5,9]$. One meta-analysis showed that the pooled prevalence of both gonorrhea and chlamydia was significantly higher among IBM than NIBM [6], but the prevalence of HIV was not found significantly different between above two groups by recent surveys $[8,10,12,13]$. Hence, there is an urgent need for longitudinal studies on biological measures of HIV and other STDs between IBM and NIBM, to confirm the real impact of Internet socializing of MSM on their HIV infection risk [46].

The subtypes of HIV are quite diverse in the MSM population, with the subtypes correlating with geographic location, transmission routes, and even sexual characteristics [14-16]. HIV bioinformatics analysis has been used to elucidate the transmission origin, transmission routes, and evolutionary dynamics of HIV in MSM and other populations [17]. However, scarce information is available about the molecular characteristics of HIV in IBM.

The main objectives of this study are to investigate the dynamics of MSM seeking homosexual partners through the Internet, the prevalence of HIV and its trends over time among IBM and NIBM, factors correlating with HIV infection among IBM and NIBM, and the difference in HIV subtypes and HIV transmitted drug resistance (TDR) rates between IBM and NIBM in Shenyang, China.

\section{Materials and Methods}

2.1. Study Design. This study was conducted in the city of Shenyang in China. Shenyang is the provincial capital of Liaoning Province and one of the largest cities in China, with a population of approximately 8.1 million. Shenyang serves as the commercial and transportation hub of China's northeast region, with frequent trade between Russia, Japan, and Korea.

A six-year serial cross-sectional study was conducted in Shenyang, China, from February 2009 to December 2014. The Institutional Review Boards of the First Affiliated Hospital of CMU approved the study protocol and informed consent forms.

2.2. Study Participant Enrollment. The Voluntary Counseling and Testing (VCT) clinic of the First Affiliated Hospital of China Medical University (CMU) in Shenyang was chosen as the study site. The VCT clinic provides the largest free HIV testing and counseling service in Shenyang. Demographics, types, and numbers of sexual partners and high-risk HIV behaviors were collected from MSM who came to the VCT clinic for the first time. Fingerprinting was used as identification for participants. Eligible entry criteria were (1) being biologically male, (2) at least 18 years old, (3) self-reported as having anal or oral sex with another male in the past 12 months, (4) self-reported as never testing positive for HIV, and (5) being able to provide written informed consent. The exclusion criteria of this study were (1) not meeting one of five entry criteria or (2) MSM who already tested positive for HIV at VCT.
2.3. Interview Process. Participants were interviewed by using a face-to-face, structured questionnaire. The following data was collected: marriage status, occupation, monthly salary, age of male sexual behavior debut, number of male sexual partners, condom use with male sexual partners, preferred sexual roles, sexual orientation, and STI symptoms in the past 12 months (pain during urination, sensation of burning in the genitals, or abnormal urethral discharge) [18]. Ways of seeking homosexual partners were collected from each subject individually; those subjects who self-reported mainly sought male sexual partners through Internet or GSN applications in past 12 months were defined as IBM, otherwise were defined as NIBM.

2.4. Laboratory Testing. Five $\mathrm{ml}$ of venous blood was drawn to test antibodies of HIV-1 and syphilis. Blood specimens positive for HIV were further tested for HIV subtypes and transmitted HIV drug resistance.

2.4.1. Antibodies to HIV-1 and Syphilis. HIV-1 antibody was first screened by enzyme-linked immune sorbent assay (ELISA) (Vironostika HIV-1 Microelisa System; BioMerieux, Durham, NC). Positive specimens were further confirmed by HIV-1/2 Western blot assay (HIV Blot 2.2 WB; Genelabs Diagnostics, Singapore).

Syphilis was tested by rapid plasma regain (RPR) (Diagnosis; Shanghai, Kehua, China), and any RPR positive samples were then tested by treponema pallidum particle assay (TPPA) (Serodia, Japan). Samples that tested positive for both RPR and TPPA were determined to be currently infected with syphilis.

2.4.2. HIV Molecular Analysis. Ribonucleic acid (RNA) was extracted to synthesize cDNA from plasma. Individual genome amplification and sequencing amplified the $3^{\prime}$ or $5^{\prime}$ halves of viral cDNA. Single sequence fragments of each amplification was combined and edited by the sequencher program (version 4.9). We used Bioedit Software Alignment followed by the Los Alamos HIV Sequence Database. Subtyping analysis was performed by the neighbor-joining method in MEGA version 4.0. The Stanford HIV Drug Resistance Database (https://hivdb.stanford.edu/) was used to analyze mutation profiles and predict genotypic resistance.

All the above laboratory tests were conducted at the Key Laboratory of AIDS and Immunology of National Health and Family Planning Commission at CMU.

2.5. Data Analysis. Questionnaires were entered using EpiData 3.1 software. Data were analyzed using SPSS (SPSS version 20.0, IL, USA). Chi-square test was used to compare differences in categorical variables. Multivariable logistic regression analysis was used to determine independent factors correlated with HIV infection. Potential confounding factors, including age, monthly income, and education level, were all adjusted in the multivariable logistic models. Trend analysis of IBM and HIV prevalence over time was performed by chi-square trend test (linear-by-linear association). $p$ value $<0.05$ was considered statistically significant in the analysis. 
2.6. Ethical Issues. A six-digit number was used to identify the participant and to link the questionnaire and laboratory test results with the participant. Written informed consent was obtained from each participant. The participants could refuse to answer any of the questions. Those who tested positive for HIV or syphilis received an immediate referral to professional HIV clinics and STI clinics. All documents with identifying information were placed in a locked file cabinet, which cannot be accessed by anyone else except for the principal investigator and study staff.

\section{Results}

3.1. Characteristics of IBM Participants. Among 1998 MSM who were approached to take part in the study, 10 refused to attend the study and 7 were excluded for had been tested HIV positive before attending the study. Among 1,981 eligible MSM, 50.5\% (1000/1981) reported using the Internet as their main method for seeking homosexual partners. Compared with NIBM subjects, IBM subjects were younger, had more proportion of local residents, had a high school level education or above, and earned a higher income (each $p<$ 0.05). IBM participants had more proportion of homosexual orientation, earlier age of male sexual behavior debut, used condoms more frequently, and more preferred receptive anal sexual roles (each $p<0.05$ ). But the ethnicity and the number of homosexual partners in the past year had no statistical difference between IBM and NIBM populations (Table 1).

\subsection{Factors Correlated with Internet-Based Sex-Seeking Behav-} ior. After adjusting for age, monthly incomes, and level of education, we found that IBM was associated with having Shenyang city as a place for residence $(\mathrm{aOR}=1.2,95 \% \mathrm{CI}$ $[1.0-1.5], p=0.040)$, being single $(\mathrm{aOR}=2.0,95 \% \mathrm{CI}[1.6-$ 2.5], $p<0.001$ ), having anal intercourse at age of 25 years or younger (less than 18 years [aOR $=2.5,95 \% \mathrm{CI}[1.7-3.6]$, $p<0.001]$; between 18 and 25 years $[\mathrm{aOR}=1.9,95 \% \mathrm{CI}$ [1.4-2.6], $p<0.001]$ ), preferring receptive anal intercourse $(\mathrm{aOR}=1.5,95 \% \mathrm{CI}[1.1-2.0], p=0.004)$, being of homosexual orientation ( $\mathrm{aOR}=1.3,95 \% \mathrm{CI}$ [1.1-1.6], $p=0.006)$, and having STD symptoms in the past year $(\mathrm{aOR}=1.6,95 \% \mathrm{CI}$ [1.0-2.6], $p=0.036$ ) (Table 1).

\subsection{HIV/Syphilis Prevalence and Molecular Characteristics.} Between 2009 and 2014, the proportion of IBM among total recruited MSM showed a rapid increased trend (from 43.3\% to $61.5 \%$, trend $p<0.001$ ). Between 2009 and 2010, the increment of HIV prevalence among IBM (from 5.7\% to 12.5\%) and NIBM (from 7\% to 13.5\%) was similar. While, from 2010 to 2014, the increment of HIV prevalence among IBM was more rapid among IBM (from $12.5 \%$ to $20.7 \%$, trend $p<$ 0.001 ) than NIBM (from $13.5 \%$ to $14.7 \%$, trend $p=0.014$ ) (Figure 1).

HIV-1 nucleotide sequences were amplified and sequenced from drawn HIV positive specimens during 2012 to 2014 , in which $93.9 \%$ (123/131) were successfully amplified. The proportion of HIV subtypes had no statistical difference between the IBM group and the NIBM group, although

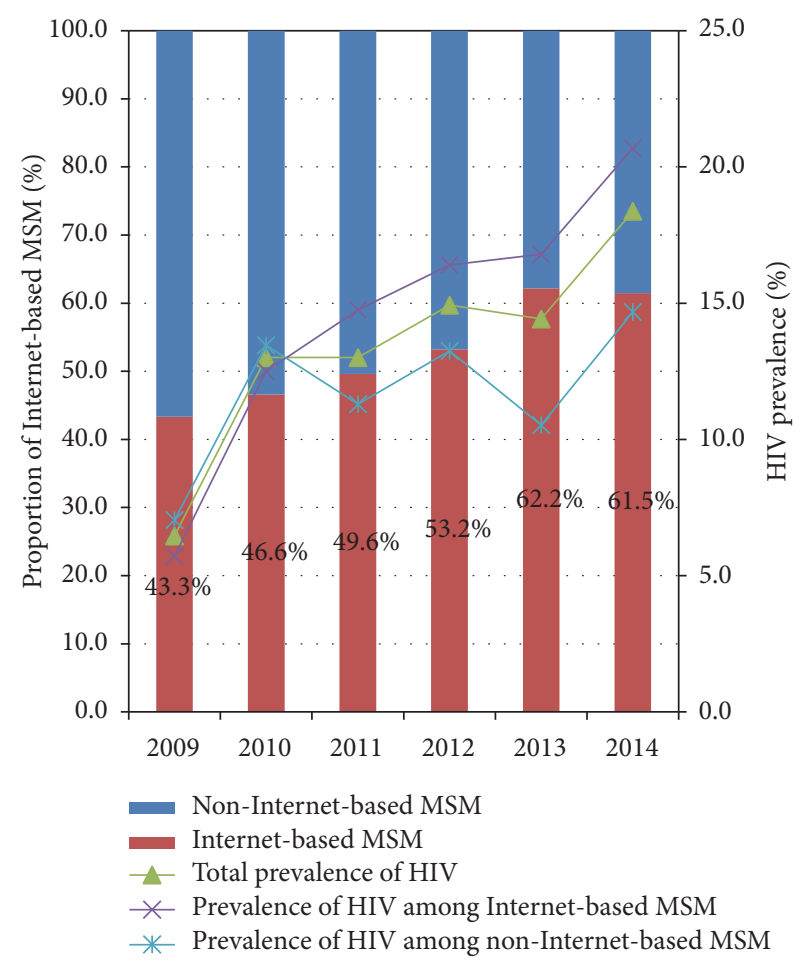

FIGURE 1: The annual prevalence of HIV among Internet-based MSM was 5.7\% (18/315), 12.5\% (17/136), 14.8\% (9/61), 16.4\% (31/189), $16.8 \%(21 / 125)$, and $20.7 \%(36 / 174)$. And the annual prevalence of HIV among non-Internet-based MSM was 7.0\% (29/412), $13.5 \%$ (21/156), $11.3 \%$ (7/62), $13.3 \%$ (22/166), $10.5 \%$ (8/76), and $14.7 \%$ $(16 / 981)$.

the former had slightly lower proportion of CRF01_AE (72.2\% versus $79.5 \%, p=0.520)$. The total proportion of TDR (5.1\% versus $6.8 \%, p=0.487)$, protease inhibitors (PIs) resistance rate $(3.8 \%$ versus $2.3 \%, p=0.549)$ and nonnucleoside reverse-transcriptase inhibitors (NNRTIs) resistance rate $(1.3 \%$ versus $4.5 \%, p=0.291)$ also had no statistical difference between the two groups (Tables 2 and $3)$.

3.4. Associated Factors between HIV Infection and Internet Use to Seek Partners. Multivariable logistic analysis showed that, after adjusting for age, monthly income, and education level, MSM who sought partners through the Internet had significantly higher odds of HIV infection compared to MSM who did not use the Internet to seek partners $(\mathrm{aOR}=1.4,95 \%$ CI [1.0-1.9], $p=0.032$ ) (Table 4).

Among the IBM population, the independent and statistically significant $(p<0.05)$ risk factors correlated with HIV infection included not being a resident of Shenyang (aOR,1.9), occasionally or never using condoms with steady male partners and casual male partners (aOR,1.9 and aOR,1.7, resp.), preferring both insertive and receptive anal sexual roles $(\mathrm{aOR}, 2.0)$, preferring only receptive anal sexual role $(\mathrm{aOR}, 2.5)$, and testing positive for syphilis $(\mathrm{aOR}, 4.3)$ (Table 4). 


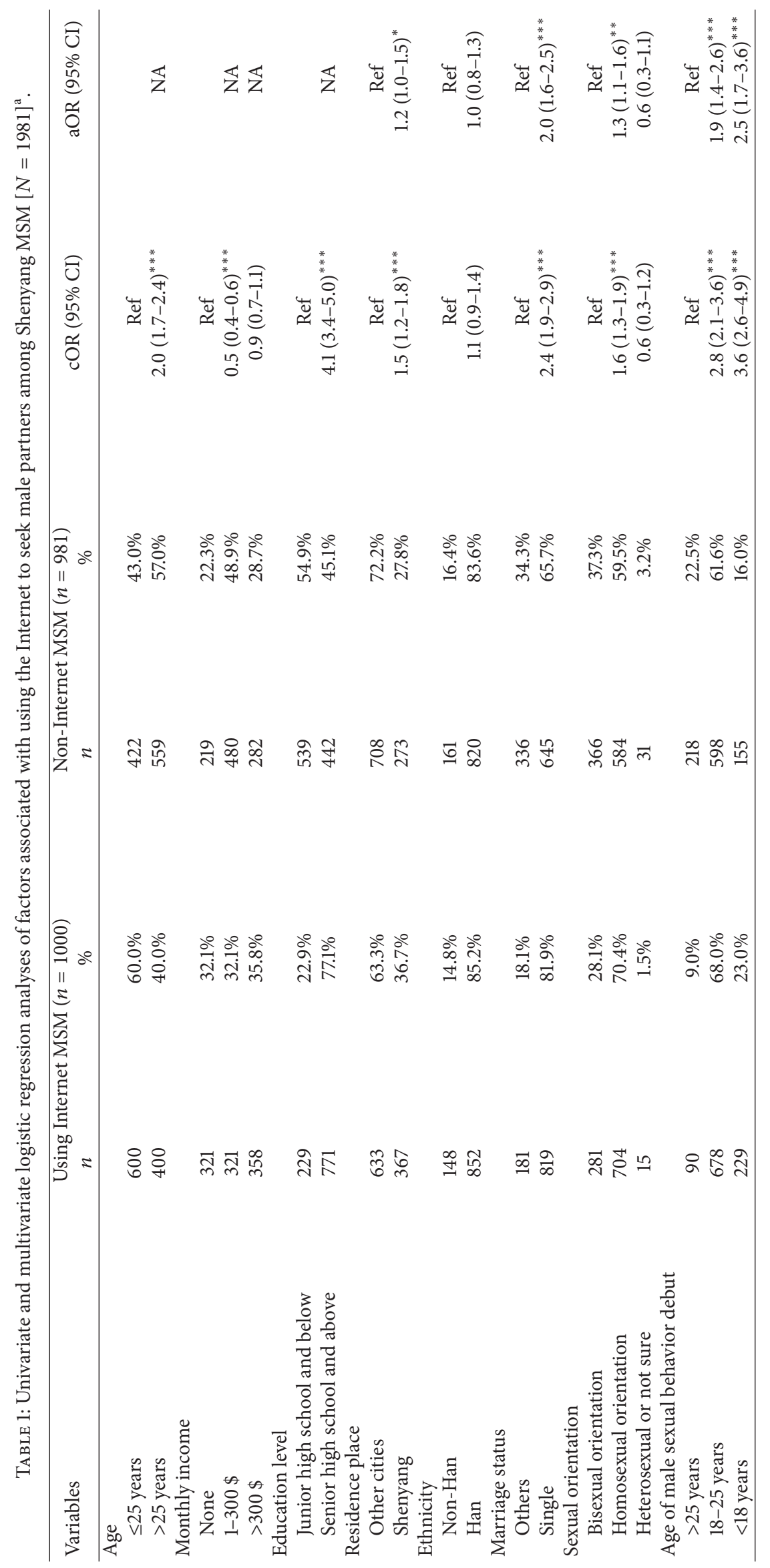




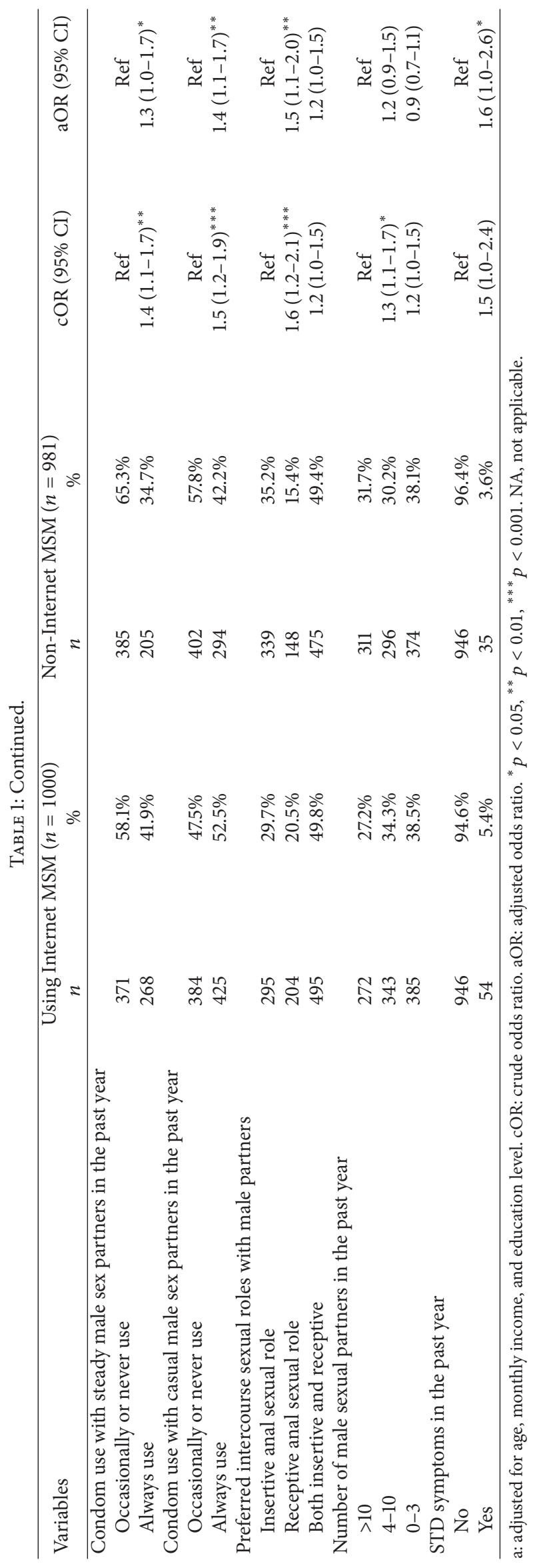


TABLE 2: HIV/syphilis prevalence and molecular characteristics of HIV of Shenyang MSM from 2009 to 2014 [ $N=1981]$.

\begin{tabular}{|c|c|c|c|c|c|c|}
\hline \multirow{2}{*}{ Variables } & \multicolumn{2}{|c|}{ Using Internet MSM } & \multicolumn{2}{|c|}{ Non-Internet MSM } & \multirow{2}{*}{ Total } & \multirow{2}{*}{$\%$} \\
\hline & $n$ & $\%$ & $n$ & $\%$ & & \\
\hline \multicolumn{7}{|c|}{ Syphilis infection } \\
\hline Positive & 114 & $11.4 \%$ & 118 & $12.0 \%$ & 232 & $11.7 \%$ \\
\hline Negative & 886 & $88.6 \%$ & 863 & $88.0 \%$ & 1749 & $88.3 \%$ \\
\hline \multicolumn{7}{|c|}{ HIV infection } \\
\hline Positive & 132 & $13.2 \%$ & 103 & $10.5 \%$ & 235 & $11.9 \%$ \\
\hline Negative & 868 & $86.8 \%$ & 878 & $89.5 \%$ & 1746 & $88.1 \%$ \\
\hline
\end{tabular}

TABLE 3: Molecular characteristics of HIV of Shenyang MSM from 2012 to 2014 [ $N=123$ ].

\begin{tabular}{|c|c|c|c|c|c|c|}
\hline \multirow{2}{*}{ Variables } & \multicolumn{2}{|c|}{ Using Internet MSM } & \multicolumn{2}{|c|}{ Non-Internet MSM } & \multirow{2}{*}{ Total } & \multirow{2}{*}{$\%$} \\
\hline & $n$ & $\%$ & $n$ & $\%$ & & \\
\hline \multicolumn{7}{|l|}{ Subtype } \\
\hline CRF01_AE & 57 & $72.2 \%$ & 35 & $79.5 \%$ & 92 & $74.8 \%$ \\
\hline CRF07_BC & 9 & $11.4 \%$ & 5 & $11.4 \%$ & 14 & $11.4 \%$ \\
\hline Other subtypes & 13 & $16.5 \%$ & 4 & $9.1 \%$ & 17 & $13.8 \%$ \\
\hline \multicolumn{7}{|l|}{ Drug resistance } \\
\hline Yes & 4 & $5.1 \%$ & 3 & $6.8 \%$ & 7 & $5.7 \%$ \\
\hline No & 75 & $94.9 \%$ & 41 & $93.2 \%$ & 116 & $94.3 \%$ \\
\hline \multicolumn{7}{|l|}{ PIs resistance rate } \\
\hline Yes & 3 & $3.8 \%$ & 1 & $2.3 \%$ & 4 & $3.3 \%$ \\
\hline No & 76 & $96.2 \%$ & 43 & $97.7 \%$ & 119 & $96.7 \%$ \\
\hline \multicolumn{7}{|c|}{ NNRTIs resistance rate } \\
\hline Yes & 1 & $1.3 \%$ & 2 & $4.5 \%$ & 3 & $2.4 \%$ \\
\hline No & 78 & $98.7 \%$ & 42 & $95.5 \%$ & 120 & $97.6 \%$ \\
\hline
\end{tabular}

Among the NIBM population, the independent and statistically significant $(p<0.05)$ risk factors correlated with HIV infection included being nonsingle (aOR,1.7), occasionally and never using condoms with steady male partners (aOR,3.0), preferring both insertive and receptive anal sexual role (aOR,2.3), and testing positive for syphilis $(\mathrm{aOR}, 4.7)$ (Table 4).

\section{Discussion}

To our knowledge, this is the first longitudinal study examining the relationship between Internet-based partner-seeking behavior and HIV infection risk. Our study expanded on the recently published literature on Internet-based partnerseeking behavior of MSM and confirmed that the IBM population had higher HIV prevalence than the NIBM population. Furthermore, the HIV prevalence in the IBM population increased more rapidly than NIBM over a 5-year time period. Our findings indicate that using the Internet to seek partners and sex is associated with an increased HIV infection and transmission risk and is correlated with distinct virtual sexual network and HIV strains.

Previous studies have failed to find a difference in age, education, and income between IBM and NIBM populations [5]. However, four peer surveys have found that, compared to NIBM, IBM population was younger, received a higher level of education, and had higher income [4, 9-11]. Our study results confirmed the findings from the four peer surveys. Furthermore, our results show that IBM tend to be single and local residents, in study site (Shenyang city).

There was also a lack of consensus in the literature about whether the Internet can increase the number of homosexual partners and the rate of unprotected anal intercourse (UAI). Grosskopf et al. found no difference in the rate of UAI and the number of homosexual partners between IBM and NIBM $[7,10,11]$. Andovitz's survey reported that the rate of UAI was significantly lower in IBM than in NIBM [8]. However, Tang's survey showed that IBM engaged in more UAI, in addition to group sex and commercial sex. Our study found that IBM had less UAI with both steady male partners and casual male partners. In regard to number of homosexual partners, Phillips [13], Lehmiller [5], and Tang [4]'s surveys indicated that IBM subpopulation had a greater number of homosexual partners. Our findings showed that the number of homosexual partners did not differ between IBM and NIBM. One possible explanation over our findings' discrepancies with previous studies' findings is that this study used venue-based recruitment method, while most of the above surveys used Internet or mobile application-based methods.

It is worth mentioning that this serial cross-sectional survey found that IBM had a statistically significant higher HIV prevalence than NIBM after adjusting for potential study confounders. We found IBM preferred receptive anal sexual roles and that more STD symptoms occurred in IBM. 


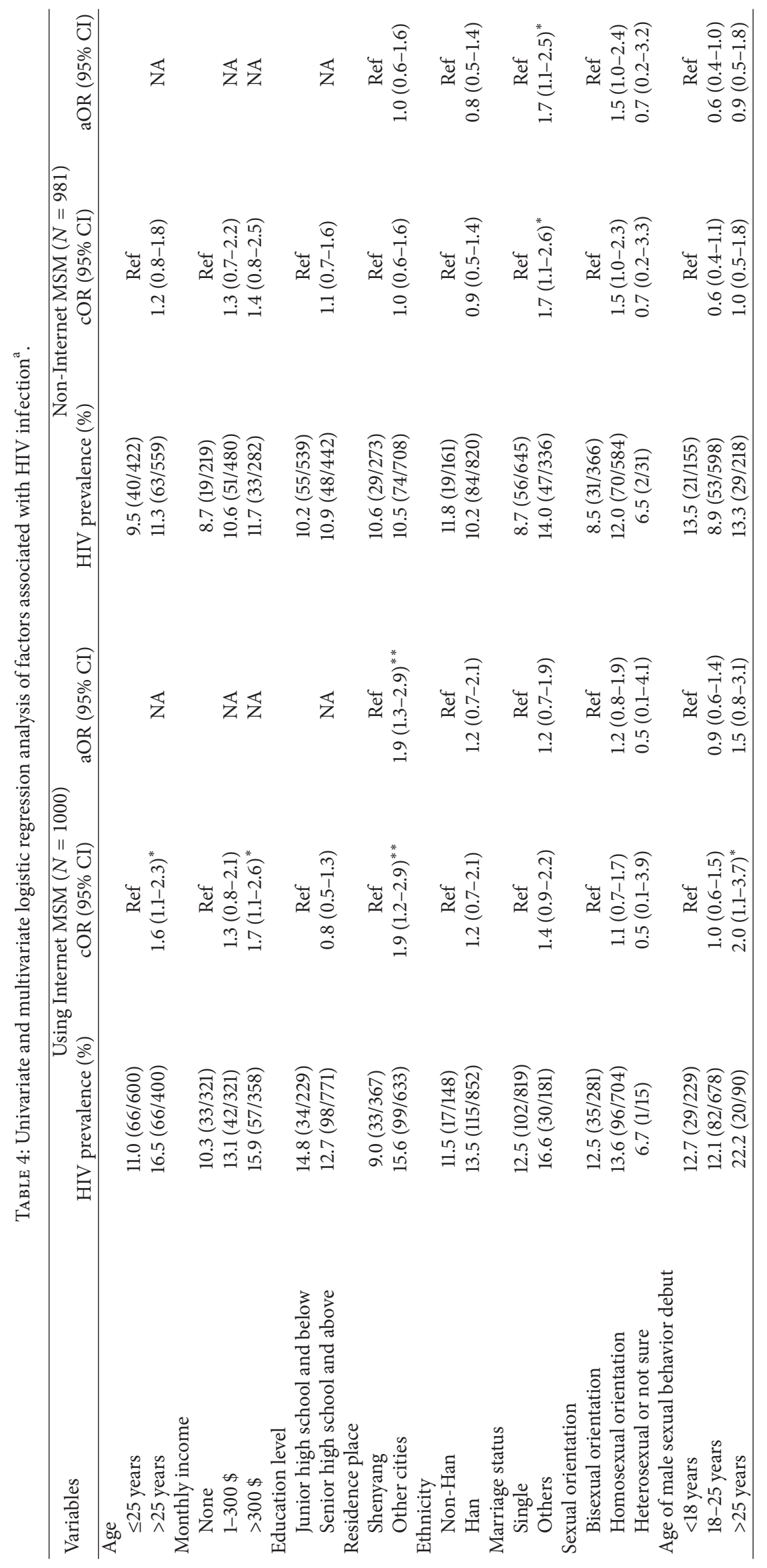




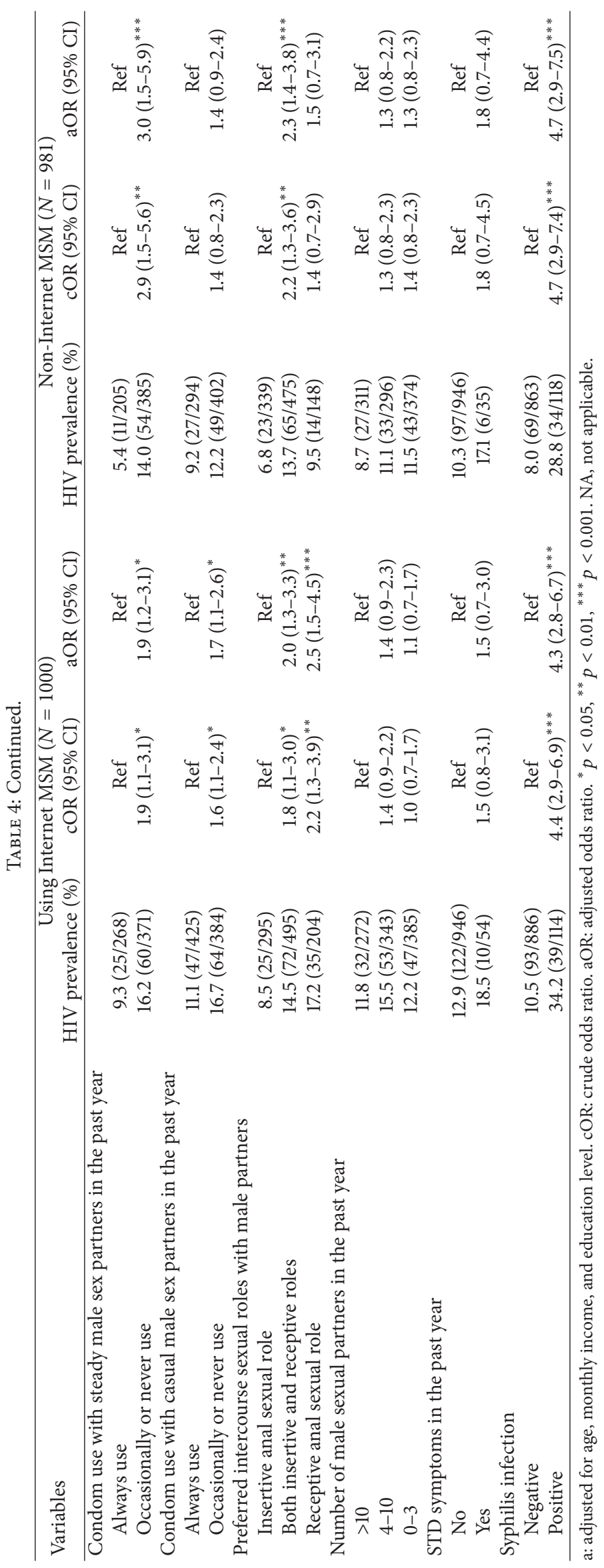


Both of these factors can significantly increase IBM's risk for acquiring HIV [19-22]. This study also was the first to show that there was a great increase in the Internet seeking behavior in Shenyang during the six-year survey, in which the proportion of IBM increased from $43.3 \%$ to $61.5 \%$. Moreover, our study also found a changing trend in the prevalence of HIV in IBM compared to NIBM. The year of 2010 was a boundary time point. Before 2010, the prevalence of HIV among IBM was slightly lower than NIBM. But from 2010 to 2014, the HIV prevalence rate in IBM increased from $12.5 \%$ to $20.7 \%$, while the HIV prevalence in NIBM did not have a significant change (from $13.5 \%$ to $14.7 \%$ ). Compared to previous studies on the relationship of Internet-based partner-seeking behavior and HIV infection risk in MSM, this study was able provide laboratory testing results as well. However, this study was conducted in MSM who sought HIV counseling and testing in one big health center of Shenyang city. More longitudinal studies are needed to further examine the relationship of Internet-based partner-seeking behavior of MSM and HIV infection risk.

In the setting of continuous implementation of large scale of free antiviral therapy, the HIV TDR rate of MSM increased from $3.8 \%$ in 2004 to $4.7 \%$ in 2014 [23, 24]. This study revealed that the HIV TDR rate of Shenyang MSM is all higher than the national rate of MSM (total TDR rate, $5.7 \%$ versus $3.6 \%$; PIs resistance rat, $3.3 \%$ versus $1.7 \%$ NNRTIs resistance rat, $2.4 \%$ versus $0.5 \%$ ) [25]. The majority of HIV TDR and subtypes are CRF01_AE and PIs in Shenyang MSM, which was similar to previous study results in the same city [26]. Our study failed to found statistical differences between IBM and NIBM subpopulations in HIV TDR rates and HIV subtypes, although slightly fewer HIV CRF01_AE subtypes existed in IBM in contrast with NIBM (72.2\% versus $79.5 \%)$. It showed that currently the Internet-based partner-seeking behavior have not substantial impact for their distribution of HIV subtypes and TDR. And the statistical power to determine a difference in HIV TDR and subtypes may be relatively lower due to the relatively small sample size of HIV positive subjects. Hence, HIV TDR and subtypes of IBM and NIBM should be continually monitored to provide molecular biomarker proof about transmission routes and clustering characteristics of IBM subpopulation.

We found similar independent risk factors for HIV infection in both IBM and NIBM populations, which were syphilis infection, preferring both receptive and insertive anal intercourse with steady male partners, and occasionally or never using condoms with steady sexual partners. These HIV infection risk factors were also reported by previous studies in MSM $[17,20,27,28]$. Our findings indicate that public health officials should pay attention to relevant contextual factors that may promote HIV infection, including UAI with homosexual partners and concurrent STD infection. In addition, HIV/AIDS health education and condoms should be provided to MSM groups who prefer receptive anal role in order to raise their condom use and negotiation skills with their homosexual partners $[29,30]$.

We also found some distinct independent HIV infection risk factors in IBM. Among the IBM population, migrant MSM had a higher HIV prevalence than local MSM. Migrant
MSM are more likely to engage in higher risk sexual risk behaviors and had lower level of HIV/AIDS knowledge [31]. They are also more likely to be the victims of sexual violence [32]. Moreover, they might lack knowledge about the availability of free HIV testing sites [31,33]. As a result, HIV/AIDS intervention programs should be targeted towards migrant MSM, in particular those who seek partners through the Internet.

Our findings also show that IBM who occasionally or never use condoms with casual male sexual partners had significantly higher HIV prevalence. Many of these IBM will use Internet applications designed to help MSM to find casual sexual partners [5]. Thus, the combination of UAI with casual sexual partners has a great impact on HIV infection risk for IBM. To increase IBM's condom use with casual partners, more HIV/AIDS knowledge should be provided, particularly about the long asymptomatic period of HIV infection and the fact that HIV positive individuals can appear healthy.

Several strengths exist in this study. First, it is one of the few venue-based surveys about the relationship between Internet-based partner-seeking behavior of MSM and HIV infection. Thus, the HIV prevalence data was more reliable and accurate than self-reported HIV/STI testing results $[5,8$, $10,13,34]$. Previously only one study reported lab-confirmed HIV/STD infections [9]. Second, this is the first serial crosssectional study, which can provide stronger evidence about trends of MSM seeking homosexual partners through the Internet. Third, this study also revealed differences in HIV subtype distribution and TDR rate between IBM and NIBM, which can help public health policy makers to understand the HIV transmission routes of Chinese IBM and provide effective and comprehensive interventions.

Some limitations to this study exist. This study used convenience sampling to recruit MSM subjects, so it cannot fully represent all of the characteristics of MSM in Shenyang. We used an interview questionnaire to collect information on some sexual behaviors, including the number of sexual partners and whether they engaged in UAI, so recall bias and social desirability bias may exist. In this survey, we did not collect the detailed frequency of using the Internet to seek sexual partners among studied subjects, and there may be some connection between the frequency and HIV infection; further studies should be conducted for future researches. We only tested for syphilis, and other STDs were not tested; more kinds of STDs should be tested to be explored in the future research to well understand the relationship between STDs and HIV infection of MSM subjects. Blood samples were not available to conduct HIV molecular testing from 2009 to 2011, so HIV subtypes and drug resistance rates derived from this study may have been different with MSM participants during that time. Despite the limitations, this study identifies the trends of Chinese MSM seeking sexual partners through the Internet and reports that Internet partner-seeking behavior is associated with significantly increased HIV prevalence. Our results have implications for behavioral interventions targeted at IBM.

Internet has become an increasingly popular means for Chinese MSM to socialize and seek partners. Our study showed that the IBM subpopulation had quite different 
demographics compared with NIBM subpopulation. The former is younger and single, with a higher educational level and a higher income. A greater proportion of IBM preferred receptive anal sexual role and STD symptoms than NIBM, which may increase their HIV infection risk. Prevalence of HIV increased more rapidly in IBM than NIBM over the course of the study. IBM also had a different distribution of HIV subtypes and TDR. More longitudinal surveys, in particular prospective cohort surveys, are needed to further examine the relationship between Internet partner-seeking behavior, sexual behaviors, and HIV infection in MSM. Gay application developers should try to integrate HIV/AIDS messaging into their platforms to promote HIV/STIs testing, condom usage, risk behavior reduction counseling, and other necessary services for the MSM community.

\section{Competing Interests}

The authors declare that there are no competing interests regarding the publication of this paper.

\section{Authors' Contributions}

Shi Pan and Jun-Jie Xu are equal contributors.

\section{Acknowledgments}

The authors thank the staff at the First Affiliated Hospital of China Medical University for their help with MSM enrollment and laboratory testing. They also thank those MSM subjects who participated in this study. They thank Dr. Alice Zhang from the University of Maryland for his assistance in manuscript editing. This study was funded by the MegaProjects of National Science Research for the 12th Five-Year Plan (2012ZX10001-006) from Dr. Hong Shang; Liaoning Educational Department Research Project (LZ2014038) from Dr. Jun-Jie Xu.

\section{References}

[1] National Health and Family Planning Commission of China, UNAIDS, and World Health Organization, "China AIDS Response Progress Report,” 2014, http://www.unaids.org/sites/ default/files/documents/CHN_narrative_report_2014.pdf.

[2] NCAIDS, NCSTD, and China CDC, "Update on the AIDS/STD epidemic in China and main response in control and prevention in December, 2015," Chinese Journal of AIDS \& STD, vol. 22, no. 2, p. 69, 2016.

[3] NCAIDS and China CDC, "Update on the AIDS/STD epidemic in China and main response in control and prevention in 2011," Chinese Journal of AIDS \& STD, vol. 18, no. 1, p. 64, 2012.

[4] W. M. Tang, B. John, Y. Zhang et al., "Gay mobile apps and the evolving virtual risk environment: a cross-sectional online survey among men who have sex with men in China," Sexually Transmitted Infections, vol. 92, no. 7, pp. 508-514, 2016.

[5] J. J. Lehmiller and M. Ioerger, "Social networking smartphone applications and sexual health outcomes among men who have sex with men," PLoS ONE, vol. 9, no. 1, Article ID e86603, 2014.
[6] H. C. Zou and S. Fan, "Characteristics of men who have sex with men who use smartphone geosocial networking applications and implications for HIV interventions: a systematic review and meta-analysis," Archives of Sexual Behavior, 2016.

[7] N. A. Grosskopf, M. T. LeVasseur, and D. B. Glaser, "Use of the Internet and mobile-based 'apps' for sex-seeking among men who have sex with men in New York City," American Journal of Men's Health, vol. 8, no. 6, pp. 510-520, 2014.

[8] R. J. Landovitz, C.-H. Tseng, M. Weissman et al., "Epidemiology, sexual risk behavior, and HIV prevention practices of men who have sex with men using GRINDR in Los Angeles, California," Journal of Urban Health, vol. 90, no. 4, pp. 729-739, 2013.

[9] M. R. Beymer, R. E. Weiss, R. K. Bolan et al., "Sex on demand: geosocial networking phone apps and risk of sexually transmitted infections among a cross-sectional sample of men who have sex with men in Los Angeles county," Sexually Transmitted Infections, vol. 90, no. 7, pp. 567-572, 2014.

[10] C. H. Bien, J. M. Best, K. E. Muessig, C. Wei, L. Han, and J. D. Tucker, "Gay apps for seeking sex partners in China: implications for MSM sexual health," AIDS \& Behavior, vol. 19, no. 6, pp. 941-946, 2015.

[11] E. R. Burrell, H. A. Pines, E. Robbie et al., "Use of the locationbased social networking application GRINDR as a recruitment tool in rectal microbicide development research," AIDS \& Behavior, vol. 16, no. 7, pp. 1816-1820, 2012.

[12] H. J. Rendina, R. H. Jimenez, C. Grov, A. Ventuneac, and J. T. Parsons, "Patterns of lifetime and recent HIV testing among men who have sex with men in New York city who use grindr," AIDS \& Behavior, vol. 18, no. 1, pp. 41-49, 2014.

[13] G. Phillips, C. Grov, and B. Mustanski, "Engagement in group sex among geosocial networking mobile application-using men who have sex with men," Sexual Health, vol. 12, no. 6, pp. 495500, 2015.

[14] J. H.-K. Chen, K.-H. Wong, K. C.-W. Chan, S. W.-C. To, Z. Chen, and W.-C. Yam, "Phylodynamics of HIV-1 subtype B among the men-having-sex-with-men (MSM) population in Hong Kong," PLoS ONE, vol. 6, no. 9, Article ID e25286, 2011.

[15] B. Zhao, X. Han, J. Xu et al., "Increase of RT-related transmitted drug resistance in non-CRF01_AE among HIV type 1-infected men who have sex with men in the 7 cities of China," Journal of Acquired Immune Deficiency Syndromes, vol. 68, no. 3, pp. 250$255,2015$.

[16] Z. Li, L. Liao, Y. Feng et al., "Trends of HIV subtypes and phylogenetic dynamics among young men who have sex with men in China, 2009-2014," Scientific Reports, vol. 5, article 16708, 2015.

[17] J. Xu, M. An, X. Han et al., "Prospective cohort study of HIV incidence and molecular characteristics of HIV among men who have sex with men(MSM) in Yunnan Province, China," BMC Infectious Diseases, vol. 13, article 3, 2013.

[18] J.-J. Xu, K. H. Reilly, C.-M. Lu et al., "A cross-sectional study of HIV and syphilis infections among male students who have sex with men (MSM) in northeast China: implications for implementing HIV screening and intervention programs," BMC Public Health, vol. 11, article no. 287, 2011.

[19] Q.-Q. Wang, X.-S. Chen, Y.-P. Yin et al., "HIV prevalence, incidence and risk behaviours among men who have sex with men in Yangzhou and Guangzhou, China: a cohort study," Journal of the International AIDS Society, vol. 17, no. 4, Article ID 18849, 2014.

[20] J.-J. Xu, M. Zhang, K. Brown et al., "Syphilis and HIV seroconversion among a 12-month prospective cohort of men who 
have sex with men in Shenyang, China," Sexually Transmitted Diseases, vol. 37, no. 7, pp. 432-439, 2010.

[21] X. Meng, H. Zou, S. Fan et al., "Relative risk for HIV infection among men who have sex with men engaging in different roles in anal sex: a systematic review and meta-analysis on global data," AIDS \& Behavior, vol. 19, no. 5, pp. 882-889, 2015.

[22] P. Pathela, K. Jamison, S. L. Braunstein, J. A. Schillinger, J. K. Varma, and S. Blank, "Incidence and predictors of HIV infection among men who have sex with men attending public sexually transmitted disease clinics, New York City, 2007-2012," AIDS \& Behavior, 2016.

[23] L. Liao, H. Xing, H. Shang et al., "The prevalence of transmitted antiretroviral drug resistance in treatment-naive HIV-infected individuals in China," JAIDS Journal of Acquired Immune Deficiency Syndromes, vol. 53, pp. S10-S14, 2010.

[24] D.-J. Liu, M.-X. Feng, and M. Liu, "Primary drug resistance of human immunodeficiency virus(HIV) among the treatmentnaïve individuals with HIV in China: a meta-analysis," Journal of Peking University (Health Sciences), vol. 47, no. 3, pp. 474-482, 2015.

[25] J. Wu and N. Wang, "The prevalence of HIV primary drug resistance among men who have sex with men in China: a metaanalysis," Chinese Journal of AIDS \& STD, vol. 20, no. 7, pp. 470473, 2014.

[26] B. Zhao, X. Han, D. Dai et al., "New trends of primary drug resistance among HIV type 1-infected men who have sex with men in Liaoning Province, China," AIDS Research \& Human Retroviruses, vol. 27, no. 10, pp. 1047-1053, 2011.

[27] W. Zhang, J. Xu, H. Zou, J. Zhang, N. Wang, and H. Shang, "HIV incidence and associated risk factors in men who have sex with men in Mainland China: an updated systematic review and meta-analysis," Sexual Health, vol. 13, no. 4, pp. 373-382, 2016.

[28] J.-J. Xu, C. Zhang, Q.-H. Hu et al., "Recreational drug use and risks of HIV and sexually transmitted infections among Chinese men who have sex with men: mediation through multiple sexual partnerships," BMC Infectious Diseases, vol. 14, no. 1, article 642, 7 pages, 2014.

[29] A. L. Herrick, S. H. Lim, C. Wei et al., "Resilience as an untapped resource in behavioral intervention design for gay men," AIDS \& Behavior, vol. 15, supplement 1, pp. S25-S29, 2011.

[30] B. T. Mausbach, S. J. Semple, S. A. Strathdee, J. Zians, and T. L. Patterson, "Efficacy of a behavioral intervention for increasing safer sex behaviors in HIV-negative, heterosexual methamphetamine users: results from the fast-lane study," Annals of Behavioral Medicine, vol. 34, no. 3, pp. 263-274, 2007.

[31] J. Wu, H. Wu, P. Li, and C. Lu, "HIV/STIs risks between migrant MSM and local MSM: a cross-sectional comparison study in China," PeerJ, vol. 4, Article ID e2169, 2016.

[32] N. He, F. Y. Wong, Z. J. Huang et al., "HIV risks among two types of male migrants in Shanghai, China: money boys vs. general male migrants," AIDS, vol. 21, supplement 8, pp. S73-S79, 2007.

[33] Y. Song, X. Li, L. Zhang et al., "HIV-testing behavior among young migrant men who have sex with men (MSM) in Beijing, China," AIDS Care, vol. 23, no. 2, pp. 179-186, 2011.

[34] H. Winetrobe, E. Rice, J. Bauermeister, R. Petering, and I. W. Holloway, "Associations of unprotected anal intercourse with Grindr-met partners among Grindr-using young men who have sex with men in Los Angeles," AIDS Care, vol. 26, no. 10, pp. 1303-1308, 2014. 


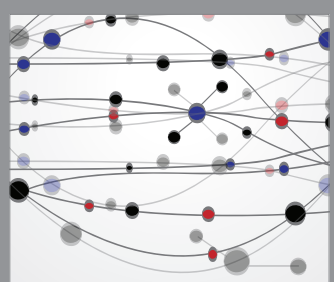

The Scientific World Journal
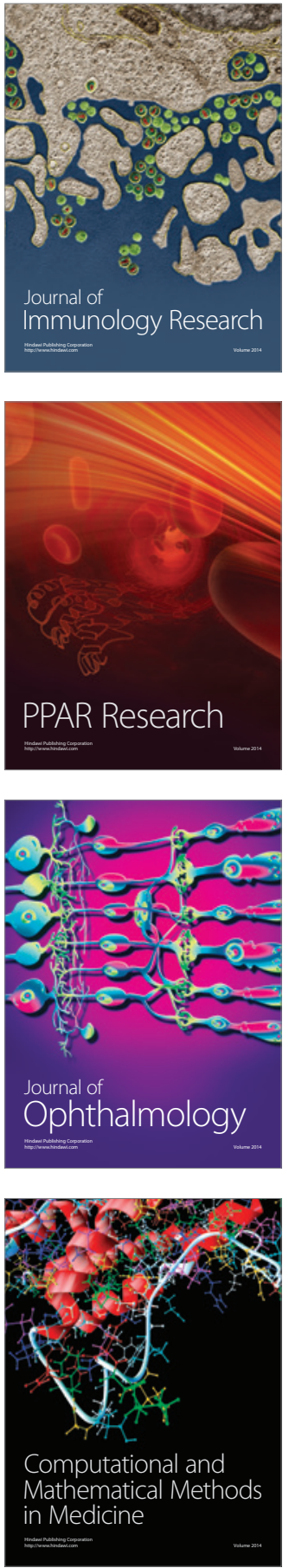

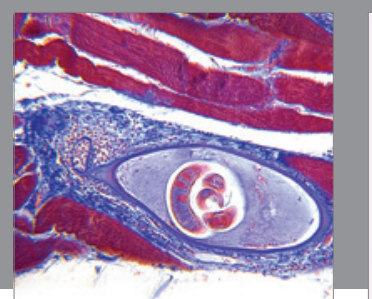

Gastroenterology Research and Practice

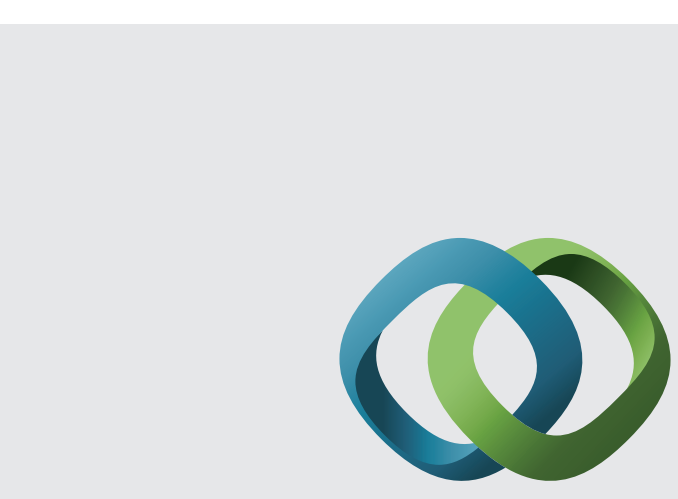

\section{Hindawi}

Submit your manuscripts at

http://www.hindawi.com
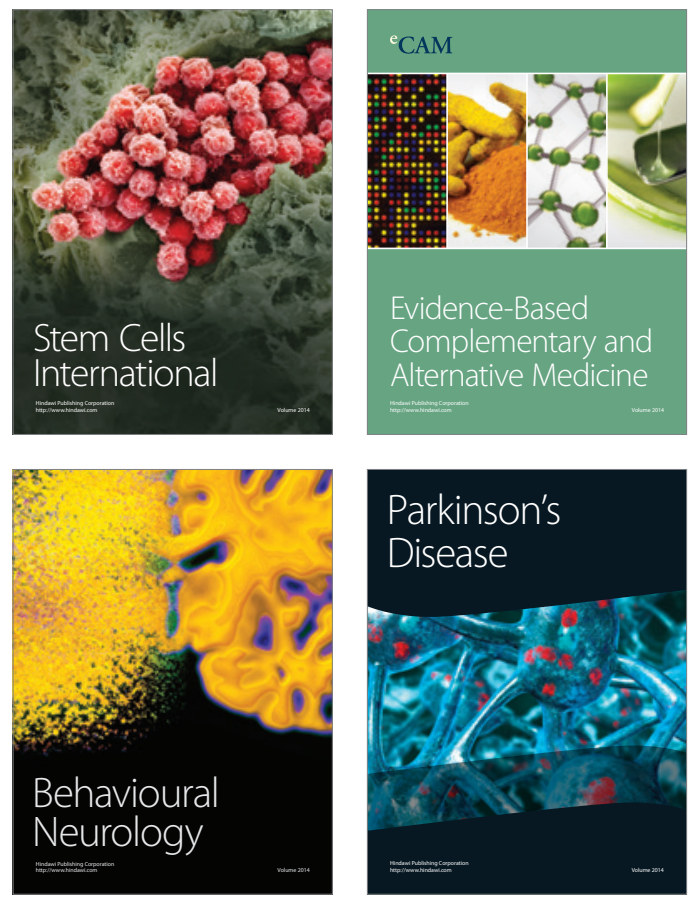
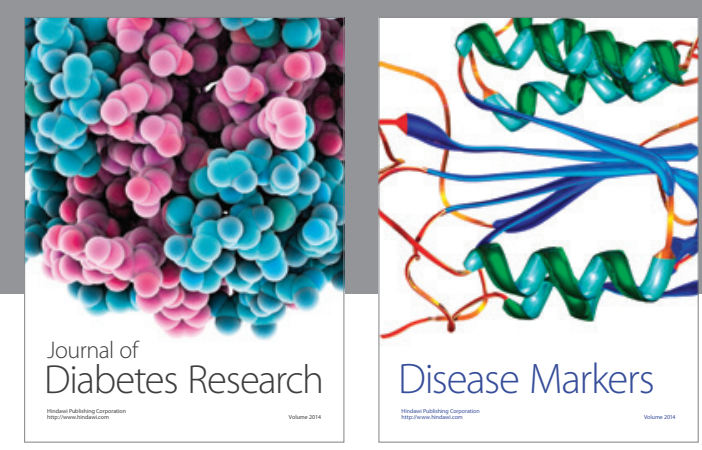

Disease Markers
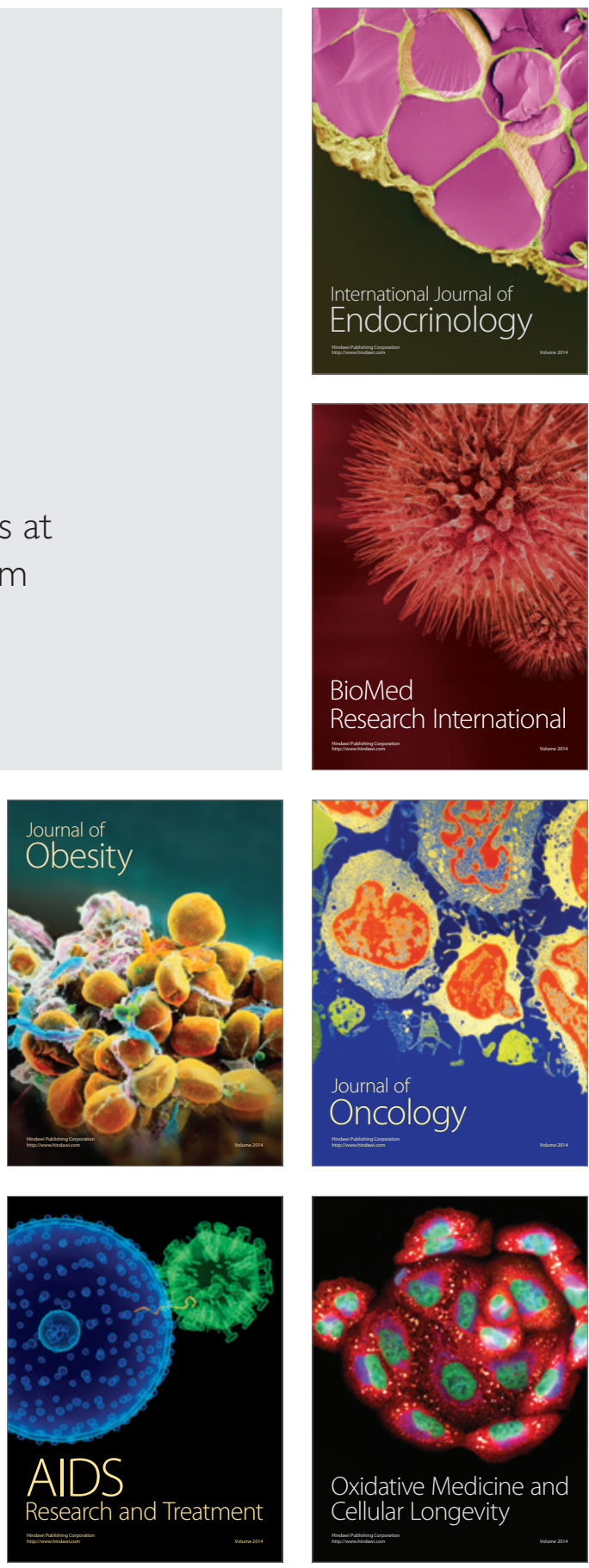